\title{
Coordinated Control of Multi-FACTS to Enhance the Dynamic Stability of the Power System
}

\author{
Rui Min ${ }^{1}$, Fei Xu' ${ }^{1}$, Fei Yuan², Zonghe Gao² \\ ${ }^{1}$ Department of Electrical Engineering, Tsinghua University, Bei Jing, China \\ ${ }^{2}$ NARI Technology Development Co., Ltd, Nanjing, China
}

Received February, 2013

\begin{abstract}
This article introduces a FACTS coordinated control strategy with impedance/admittance measurement feedback. Then the effectiveness of this method is proved in mathematics with damp torque method. The control strategy effect is verified in a single machine infinite bus system and a four machine power system with PSASP6.26 (Power System Analysis Software Package). This coordinated control strategy has practical significance to improve system dynamic stability and theoretical significance to improve system transient stability.
\end{abstract}

Keywords: Coordinated Control; Dynamic Stability; Measurement Feedback; SVC; TCSC; Transient Stability

\section{Introduction}

The power grid of China has entered the ultra-high voltage (UHV) period with large complicated power system. A large power grid needs more people to supervise and has more equipment to control because of its complicated structure. In addition, although the development of smart grid brings many opportunities to us, operators have more and more challenges to run the grid and avoid accidents. In this context, our smart grid needs more flexible and reliable control methods to reduce network losses, improve the flow distribution, improve the system stability, raise the level of power system damping and so on.

FACTS device can be regulated reliable with fast response. Individual FACTS device can improve the local power grid status with appropriate control strategies in a short period of time. If multiple FACTS devices in different areas can be coordinated with appropriate control strategies, the entire grid (or parts of) will have a better control results. With the widely application of FACTS devices, the coordination control problem of FACTS devices has an increasingly important position [1].

\section{FACTS Coordinated Control Strategy with Measurement Feedback}

\subsection{Coordinated Control of TCSC with SVC Admittance Inputs Signal}

The TCSC input control signal is determined by the control strategy, such as the acceleration power of the generator and active and reactive power flow of transmission lines. The most commonly scene to use TCSC is to con- trol the electromagnetic power of the series branch, which use electromagnetic power deviation as inputs. The PI controller parameters and control effect are constrained by TCSC capacity. When TCSC use conventional PI control, the system dynamic stability can be improved more [2].

Transmit the remote SVC admittance signal to TCSC inputs with measurement feedback is a way to improve the TCSC control effect. Coordinated control parameters between TCSC and SVC can be derived by the damping torque method. By appropriate adjustment of tuning parameters, allows the SVC produce a significant control effect to the dynamic stability of the system. The SVC can bring better effect to improve system dynamic stability by adjusting parameters appropriately. The TCSC with SVC additional admittance signal coordination control block diagram as shown in Figure 1:

\subsection{Coordinated Control of SVC with TCSC Impedance Inputs Signal}

The most commonly scene to use SVC is to maintain the stability of the system voltage. In addition, SVC can also be used to improve the dynamic and static stability performance of the power system and to improve grid dynamic stability and damping.

It is difficult to improve the system dynamic stability and maintain voltage stability by one SVC at the same time. If the SVC control strategy is to improve the dynamic stability of the system, it may have a negative impact on system voltage stability. The system transient stability should have a more significant improvement by 
using SVC conventional constant voltage proportional control.

Transmit the remote TCSC impedance signal to SVC inputs with measurement feedback is a way to improve the SVC control effect. Coordinated control parameters between SVC and TCSC can be derived by the damping torque method. By appropriate adjustment of tuning parameters, allows the TCSC produce a significant control effect to the transient stability of the system. The TCSC can bring better effect to improve system transient stability by adjusting parameters appropriately. The SVC with TCSC additional impedance signal coordination control block diagram as shown in Figure 2:

\section{Coordinated Control Affects on System Stability}

\subsection{SVC with TCSC Additional Impedance Signal}

A single machine infinite bus system is shown in Figure 3. A TCSC is installed in a transmission line between bus

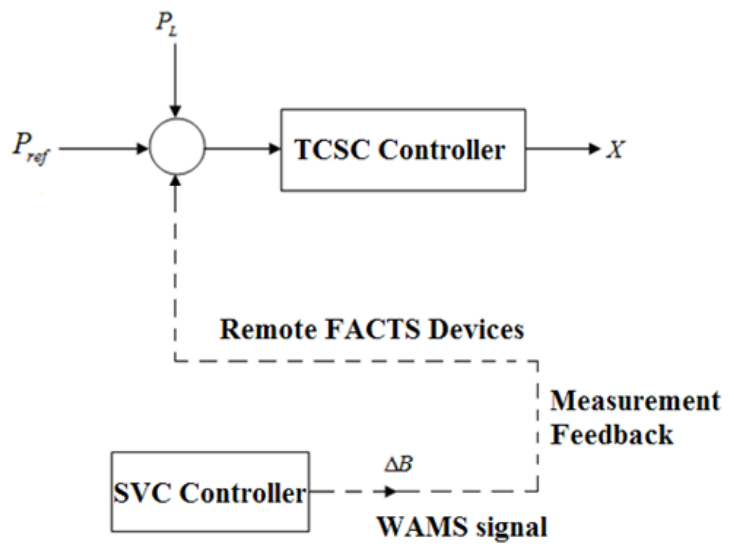

Figure 1. TCSC with SVC additional admittance signal coordination control block diagram.

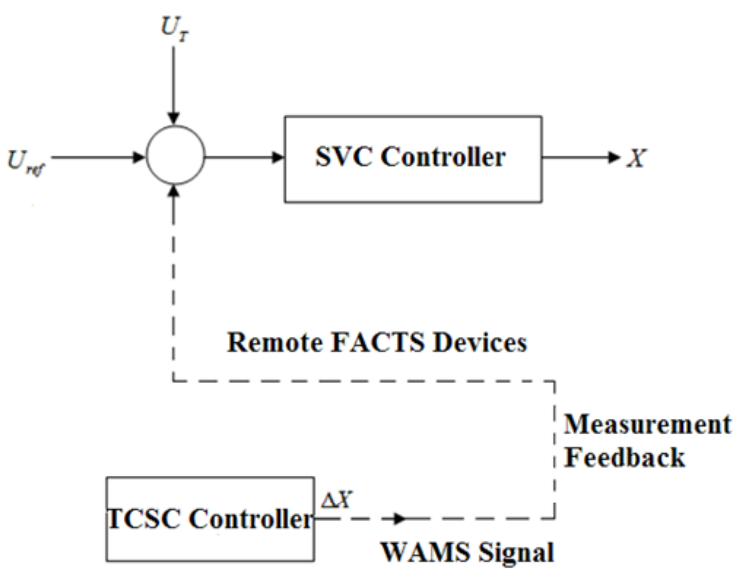

Figure 2. SVC with TCSC additional impedance signal coordination control block diagram.
2 and bus 3. A SVC is installed at bus 2. TCSC use one order PI control and damping control. SVC uses one order gain-delay process. When Studying the SVC affects to system dynamic stability, SVC and TCSC using closed-loop control and the equivalent impedance is not a constant value.

The equivalent circuit of the system is shown in Figure 4:

The generator electromagnetic power is:

$$
P=\frac{E^{\prime} U}{X_{1}+\frac{X_{2}\left(X_{C}+X_{3}\right)}{X_{2}+X_{C}+X_{3}}-B X_{1}\left(\frac{X_{2}\left(X_{C}+X_{3}\right)}{X_{2}+X_{C}+X_{3}}\right)} \sin \delta
$$

The values on the equation above are per unit values which have the following meanings:

$P$ : Generator electromagnetic power

$E^{\prime}$ : Generator transient electromotive force

$U$ : Infinite bus terminal voltage

$X_{1}$ : Sum of transformer equivalent impedance, generator transient reactance and line reactance

$X_{C}$ : The equivalent impedance of TCSC

$X_{2}$ : The equivalent impedance of the line 2

$X_{3}$ : The equivalent impedance of the line 3

$B$ : SVC equivalent admittance

$\delta$ : Generator rotor angle (radians)

$U_{B}$ : SVC parallel bus voltage

The generator uses the classic third-order model. $E_{q}^{\prime}$, $\omega$ and $\delta$ are Variables. SVC parallel bus voltage is:

$$
U_{B}=\frac{\sqrt{\left(X E^{\prime}\right)^{2}+\left(X_{1} U\right)^{2}+2 X_{1} X E^{\prime} U \cos \delta}}{X+X_{1}-B X_{1} X}
$$

In which $X=\frac{X_{2}\left(X_{C}+X_{3}\right)}{X_{2}+X_{C}+X_{3}}$

The equation (1) can be linearized to:

$$
\Delta P=K_{1} \Delta \delta+K_{2} \Delta B+K_{3} \Delta X_{C}
$$

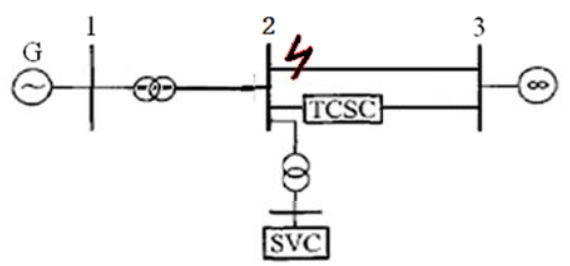

Figure 3. The Single machine infinite system.

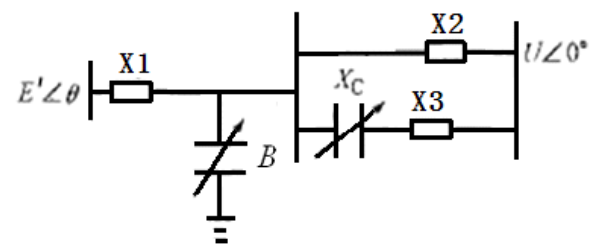

Figure 4. The Single machine infinite equivalent circuit diagram. 
In which

$$
\begin{gathered}
K_{1}=\frac{E^{\prime} U}{X+X_{1}-B X X_{1}} \cos \delta>0 \\
K_{2}=\frac{E^{\prime} U X X_{1}}{\left(X+X_{1}-B X X_{1}\right)^{2}} \sin \delta>0 \\
K_{3}=\frac{E^{\prime} U X^{\prime}\left(B X_{1}-1\right)}{\left(X+X_{1}-B X X_{1}\right)^{2}} \sin \delta<0
\end{gathered}
$$

In which $X^{\prime}=\frac{X_{2}^{2}}{\left(X_{2}+X_{C}+X_{3}\right)^{2}}$

The equation (1) can be linearized to:

$$
\Delta U_{B}=K_{4} \Delta \delta+K_{5} \Delta B+K_{6} \Delta X_{C}
$$

In which

$$
\begin{gathered}
K_{4}=\frac{-X X_{1} E^{\prime} U \sin \delta}{\left(X_{1}+X-B X X_{1}\right) \sqrt{\left(X E^{\prime}\right)^{2}+\left(X_{1} U\right)^{2}+2 X X_{1} E^{\prime} U \cos \delta}}<0 \\
K_{5}=\frac{X X_{1} \sqrt{\left(X E^{\prime}\right)^{2}+\left(X_{1} U\right)^{2}+2 X X_{1} E^{\prime} U \cos \delta}}{\left(X_{1}+X-B X X_{1}\right)^{2}}>0 \\
K_{6}=\frac{X^{\prime} U+X^{\prime}\left(B X_{1}-1\right) \sqrt{\left(X E^{\prime}\right)^{2}+\left(X_{1} U\right)^{2}+2 X X_{1} E^{\prime} U \cos \delta}}{\left(X_{1}+X-B X X_{1}\right)}>0
\end{gathered}
$$

$K_{6}>0$ is satisfied in practical engineering conditions.

The equation of SVC with TCSC additional impedance signal is:

$$
\dot{B}=\frac{1}{T_{A}}\left[K_{A}\left(U_{b e f}-U_{B}+K_{7} \Delta X_{C}\right)-B\right]
$$

The transfer function form is:

$$
\Delta B=-\frac{K_{A}}{1+s T_{A}}\left(\Delta U_{B}+K_{7} \Delta X_{C}\right)
$$

TCSC dynamic equation with the PI control is:

$$
\Delta X_{C}=\left(K_{P}+\frac{K_{I}}{s}\right) \Delta P
$$

Form Equation (3), Equation (7), Equation (11) and Equation (12), we have:

$$
\begin{aligned}
\Delta P= & {\left[K_{1}-\frac{K_{2} K_{4} K_{A}\left(1+K_{5} K_{P} K_{A}\right)}{\left(1+K_{3} K_{5} K_{P} K_{A}\right)^{2}+\left(K_{3} K_{I} T_{A}\right)^{2}}\right.} \\
& \left.+\frac{K_{7} K_{2} K_{3} K_{4} K_{I} K_{A} T_{A} s}{\left(1+K_{3} K_{5} K_{P} K_{A}\right)^{2}+\left(K_{3} K_{I} T_{A}\right)^{2}}\right] \Delta \delta
\end{aligned}
$$

Literature [3] proposed a method to compute system synchronous torque and damping torque. If the electromagnetic torque of the system can be expressed as:

$$
\Delta T_{e}(S)=K_{e}(S) \Delta \theta(S)
$$

So that the synchronous torque coefficient damp torque coefficient are:

$$
\begin{gathered}
T_{s}(j \omega)=\operatorname{Re}\left[K_{e}(j \omega)\right] \\
T_{d}(j \omega)=(1 / \omega) \operatorname{Im}\left[K_{e}(j \omega)\right]
\end{gathered}
$$

If the changes in the disturbance occurred and quell the generator speed is negligible [4], that is $\Delta T_{e}=\frac{\Delta P_{e}}{\omega} \approx \Delta P_{e}$, Form Equation (13), Equation (14), Equation (15) and Equation (16), we have:

Form Equation (13), Equation (14), Equation (15) and Equation (16), we have:

$$
\begin{gathered}
T_{s}(j \omega)=K_{1}-\frac{K_{2} K_{4} K_{A}\left(1+K_{5} K_{P} K_{A}\right)}{\left(1+K_{3} K_{5} K_{P} K_{A}\right)^{2}+\left(K_{3} K_{I} T_{A}\right)^{2}} \\
T_{d}(j \omega)=\frac{K_{7} K_{2} K_{3} K_{4} K_{I} K_{A} T_{A}}{\left(1+K_{3} K_{5} K_{P} K_{A}\right)^{2}+\left(K_{3} K_{I} T_{A}\right)^{2}}
\end{gathered}
$$

In the two equations above, $\mathrm{K}_{1}>0, \mathrm{~K}_{2}>0, \mathrm{~K}_{3}>0, \mathrm{~K}_{4}>0$, $\mathrm{K}_{5}>0, \mathrm{~K}_{6}>0$. SVC with TCSC additional impedance signal makes the system synchronous torque coefficient increased $\Delta \mathrm{T}_{\mathrm{S}}(\mathrm{j} \omega)>0$. If $\mathrm{K}_{7}>0$, the damp torque coefficient of the system $\Delta \mathrm{T}_{\mathrm{d}}(\mathrm{j} \omega)>0$. If $\mathrm{K}_{7}<0$, the damp torque coefficient of the system $\Delta \mathrm{T}_{\mathrm{d}}(\mathrm{j} \omega)<0$. It can be concluded that SVC with TCSC additional impedance signal makes the system synchronous torque coefficient increased and contribute to improve system transient stability. When the TCSC impedance signal add to the SVC input terminal as a positive gain, this control strategy can improve system dynamic stability too.

\subsection{TCSC With SVC Additional Admittance Signal}

Similar with SVC, we still using single machine infinite bus system in Figure 3.1 and Figure 3.2. The equation of TCSC with SVC additional admittance signal is:

$$
\Delta X_{C}=\left(K_{P}+\frac{K_{I}}{s}\right)\left(\Delta P+K_{8} \Delta B\right)
$$

In which

$$
\Delta B=-\frac{K_{A}}{1+T_{A} s} \Delta U_{B}
$$

Form Equation (3), Equation (7), Equation (19) and Equation (20), we have:

$$
\begin{gathered}
T_{s}(j \omega)=\frac{K_{1} K_{2} K_{4} K_{8}\left(1-K_{3} K_{5} K_{P} K_{A}\right) \omega^{2}}{\left(1-K_{3} K_{5} K_{P} K_{A}\right)^{2}+\left(K_{3} K_{I} T_{A}\right)^{2}} \\
T_{d}(j \omega)=-\frac{K_{1} K_{2} K_{4} K_{I} K_{A} T_{A}}{\left(1-K_{3} K_{5} K_{P} K_{A}\right)^{2}+\left(K_{3} K_{I} T_{A}\right)^{2}}
\end{gathered}
$$

In the two equation above, $K_{1}>0, K_{2}>0, K_{3}<0$, $K_{4}<0, K_{5}>0, K_{6}>0$. TCSC with SVC additional 
admittance signal makes the system damp torque coefficient increase $\Delta T_{d}(j \omega)>0$. If $K_{8}>0$, the synchronous torque coefficient of system. If $K_{8}<0$, the synchronous torque coefficient of the system $\Delta T_{s}(j \omega)>0$. It can be concluded that TCSC with SVC additional admittance signal makes the system damp torque coefficient increased and contribute to improve system dynamic stability. When the SVC admittance signal adds to the TCSC input terminal as a negative gain, this control strategy can improve system transient stability too.

\section{Simulation Example}

\subsection{Verification of Coordinated Control Improving System Dynamic Stability}

Single machine infinite bus system is shown in Figure 3. The three phase's short circuit fault occurs at $1.0 \mathrm{~s}$ and continued $0.1 \mathrm{~s}$ between bus 2 and bus 3 . The transmission line is recovered at 1.1s. This article uses PSASP6.26 to simulate the system. Set $K_{7}=0.5, K_{8}=-0.11$.

It can be seen form Figure 5 and Figure 6 that compared to conventional control, coordinated control improves the system dynamic stability.

The original parameters of the four-machine system specific can be seen in [5]. In this article, the two AC lines between bus 8 and bus 9 are combined into one line. Single line figure of the system is shown in Figure 7:

SVC is paralleled on bus 8 , TCSC is serried on the line between bus 8 and bus 9 . In the process of simplifying, the one phase's short circuit fault occurs at 1.0s and continued $0.1 \mathrm{~s}$ between bus 7 and bus 8 . The transmission line is recovered at $1.1 \mathrm{~s}$. There is an impact load in bus7 which remains 2 s, the result is shown in Figure 9. This article uses PSASP6.26 to simulate the system.

It can be seen form Figure 8 that compared to conventional control; TCSC with SVC additional admittance coordinated control improves the system dynamic stability in four-machine two-region systems.

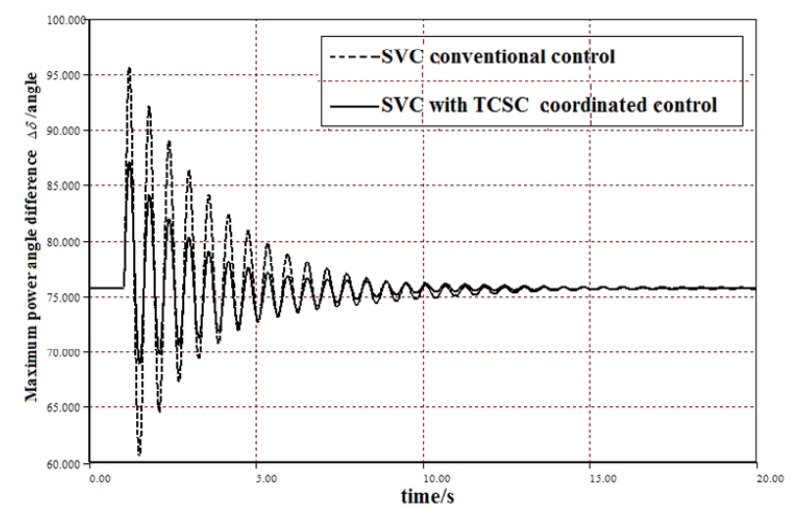

Figure 5. SVC conventional control and SVC with TCSC additional impedance coordinated control.

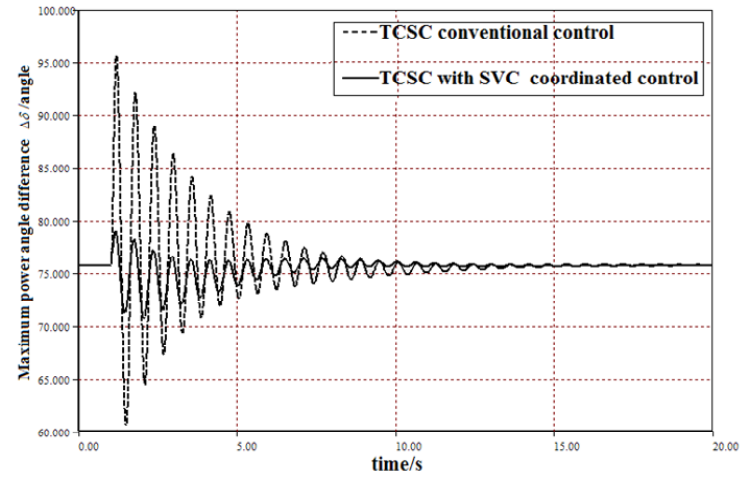

Figure 6. TCSC conventional control and TCSC with SVC additional admittance coordinated control.

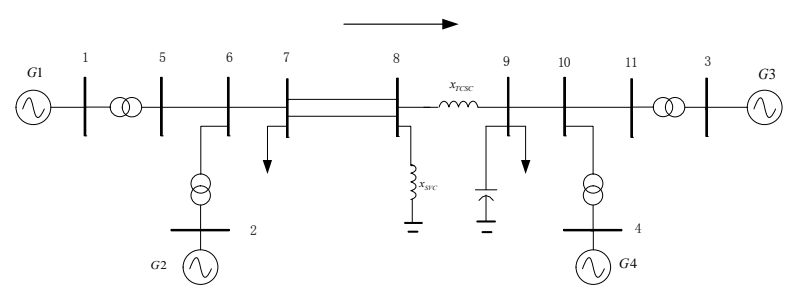

Figure 7. The single line figure of four-machine two- region systems.



Figure 8. TCSC conventional control and TCSC with SVC additional admittance coordinated control.

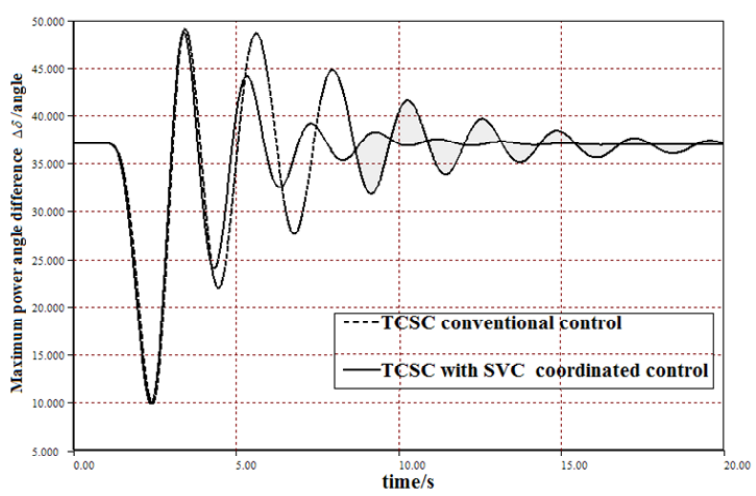

Figure 9. Impact load in TCSC conventional control and TCSC with SVC additional admittance coordinated control. 


\subsection{Verification of Coordinated Control Improving System Transient Stability}

Using the system shown in Figure $\mathbf{3}$ and Figure 7, verifying the coordinated control strategy improves the system transient stability with the same scene. The first pendulum of power angle difference and the critical clearing time are used as the transient stability evaluation index.

It can be seen form Table 1 that compared to conventional control, coordinated control improves the system transient stability.

\section{Summary}

This article introduces a FACTS coordinated control strategy with impedance/admittance measurement feedback. Then the effectiveness of this method is proved in mathematics with damp torque method. Simulation results show that the coordinated control improves system stability when, meet certain conditions.

Table 1. Transient stability evaluation index of conventional control and coordinated control.

\begin{tabular}{|c|c|c|c|}
\hline Scene & Control strategy & $\begin{array}{l}\text { first pendulum of powercriti } \\
\text { angle difference (angle) }\end{array}$ & $\begin{array}{l}\text { ical clearing } \\
\text { time (s) }\end{array}$ \\
\hline \multirow{4}{*}{$\begin{array}{l}\text { Single } \\
\text { machine } \\
\text { infinite }\end{array}$} & TCSC & 55.6 & 0.3 \\
\hline & $\begin{array}{l}\text { TCSC with SVC } \\
\text { additional signal }\end{array}$ & 49.1 & 0.43 \\
\hline & SVC & 55.6 & 0.30 \\
\hline & $\begin{array}{l}\text { SVC with TCSC } \\
\text { additional signal }\end{array}$ & 50.5 & 0.42 \\
\hline \multirow{2}{*}{$\begin{array}{c}\text { Four } \\
\text { machine }\end{array}$} & TCSC & 47.8 & 0.41 \\
\hline & $\begin{array}{l}\text { TCSC with SVC } \\
\text { additional signal }\end{array}$ & 43.9 & 0.52 \\
\hline
\end{tabular}

Because of the transient stability control has high demands on system response speed, the WAMS delay have a great adverse impact on transient stability control. So this coordinated control method has certain theoretical significance for improving the system transient stability, but currently does not have practical significance.

In dynamic stability control, some certain delay is acceptable for the overall control effect. Therefore, when the remote FACTS signals transmitted to other FACTS devices with WAMS, the system dynamic stability control can be realized in this way.

\section{Acknowledgements}

This work was supported by China's National High Technology Research and Development Program (2012AA050207), National Science and technology support program (2011BAA01B02), China's National Nature Science Foundation under Grant 51190101 and 50607011, as well as China's State Grid Technology Program and NARI Technology Program.

\section{REFERENCES}

[1] Y. J. Cao and J. Tao, "Research Progress on Interaction and Coordinated Control among FACTS Controllers," Proceedings of the CSU- EPSA, Vol. 20, No. 1, 2008, pp. 1-8.

[2] T. Ye, "Research on Connrdinated Control of Multi FACTS based on WAMS," Tsinghua University.

[3] M. Parniani and M. R. Iravani, "Voltage Control Stability and Dynamic Interaction Phenomena of Static Var Compensators," IEEE Transactions on Power Systems, Vol. 10, No. 3, 1995, pp. 1592-1597. doi:10.1109/59.466483

[4] J. Liu, X. Y. Li and G. F. Tang, "Interrelations between SVC Voltage Control and Damping Control," Proceedings of the CSEE, Vol. 28, No. 1, 2008, pp. 12-17.

[5] P. S. Kundur, "Power Systems Stability and Control (in Chinese),” Beijing, 2001, p. 548. 